\title{
PROCESSAMENTO AUDITIVO E SPECT EM CRIANÇAS COM DISLEXIA
}

\author{
Luciane Sauer ${ }^{* *}$, Liliane Desgualdo Pereira2**, \\ Sylvia Maria Ciasca ${ }^{3 *}$, Magda Pestun ${ }^{2 *}$, Marilisa M. Guerreiro ${ }^{3 *}$
}

\begin{abstract}
RESUMO - Aplicamos em um grupo de 36 crianças os testes dicótico de dígitos, dicótico de dissílabos alternados e dicótico não-verbal, os quais fazem parte do conjunto de avaliação do processamento auditivo. As crianças foram divididas em dois grupos que foram correlacionados: grupo experimental (GE) formado por 18 crianças com diagnóstico de dislexia e 18 crianças normais compondo o grupo comparação (GC), sem queixa de aprendizagem e pareadas em relação a sexo, lateralidade e nível sócio-econômico ao GE. Realizamos o exame de imagem (SPECT) no GE. Encontramos em nosso estudo diferença estatisticamente significante e nt reos grupos (GE e GC) em todos os testes avaliados. Em relação ao SPECT, apesar de não encontrarm os diferença estatisticamente significante entre os resultados, pudemos observar que $50 \%$ dos sujeitos avaliados a presentaram alteração, sendo a maior parte em áreas do lobo temporal esquerdo. Concluímos que crianças com dislexia apresentam alterações do processamento neurológico central que podem ser detectadas tanto em testes específicos de processamento auditivo, quanto em exames funcionais de imagem como SPECT.
\end{abstract}

PALAVRAS-CHAVE: dislexia, processamento auditivo, neuroimagem, teste dicótico.

\section{Dichotic listening and SPECT in dyslexic children}

\begin{abstract}
Dichotic listening (DL) was evaluated in 36 children with: verbal dichotic listening test, alternating dissilable dichotic test and non-verbal dichotic listening test. Children were separated into two groups: experimental group with 18 dyslexic children and control group with 18 normal children. Both groups were comparable in gender, laterality and social-economic level. All dyslexic children underwent neuroimaging exam (SPECT). Our data showed that there was a statistical difference between both groups in all DL tests. Abnormal SPECT findings were seen in $50 \%$ of the dyslexic children, hypoperfusion of the left temporal lobe being the most frequent abnomality. We conclude that dyslexic children present an impairment of central neurologic processing that may be detected by DL tests, and by functional imaging exam, such as SPECT, as well.
\end{abstract}

KEY WORDS: dyslexia, imaging, dichotic listening test.

Todos os animais se comunicam, mas só o homem é capaz de falar, ler e escrever. A leitura e escrita são as formas mais elevadas da linguagem, que exigem um processo linguístico anatômico e neuropsicológico altamente complexo. A dificuldade de aprendizagem para as habilidades de leitura e escrita são denominadas de dislexia do desenvolvimento ou, segundo o DMS-IV, transtorno de leitura, que pode também coexistir com dificuldades na linguagem oral, cálculo, atenção, memória e integração perceptivo-motora'. A dislexia do desenvolvimento parece ser um distúrbio neurológico congênito, acometendo estruturas subcorticais e corticais ${ }^{1,2}$. SPECT (Single Photon Emis - sion Computed Tomography) é uma modalidade de imagem que permite avaliação funcional do cérebro, ou seja, fornece informações relacionadas ao fluxo sanguíneo cerebral regional de forma qualitativa e quantitativa e demonstra acurácia no diagnóstico de déficits neuropsicológicos estabelecidos em condições basais ${ }^{3,4}$. Recentes estudos têm utilizado o SPECT com o objetivo de localizar e comprovar a existência de déficits funcionais neurológicos, como causador de grande variedade de manifestações que levam à incapacidade de adquirir a habilidade de leitura e escrita, definida como dislexia ${ }^{4,5}$.

Katz ${ }^{6}$ relatou que crianças com distúrbio de apren-

\footnotetext{
*Departamento de Neurologia da Faculdade de Ciências Médicas da Universidade Estadual de São Paulo, Campinas SP, Brasil (FCM/Unicamp); ** Departamento de Distúrbios da Comunicação da Universidade Federal de São Paulo, São Paulo SP, Brasil (UNIFESP): 'Pós-Graduanda; '2Professora Doutora; ${ }^{3}$ Professora Associada.
}

Recebido 18 Maio 2005, recebido na forma final 25 Agosto 2005. Aceito 11 Outubro 2005.

Dra. Marilisa M. Guerre iro - Departamento de Neurologia - FCMIUnicamp - Caixa Postal 6111 - 13083-970 Campinas SP - Brasil. E-mail: mmg@fcm.unicamp.br 
dizagem podem ter distúrbios neuroauditivos. Esses distúrbios envolvem o sistema nervoso auditivo central e podem ser identificados, analisados e quantificados através da avaliação do Processamento Auditivo (PA). De acordo com a definição proposta pela Ame rican Speech-Hearing and Language Association (ASHA) ${ }^{7}$, o PA consiste de mecanismos e processos do sistema auditivo que são responsáveis por diversos fenômenos comportamentais, incluindo fala e linguagem, tendo correspondência neurofisiológica, assim como funcional. Há consideráveis evidências indicando uma relação entre os distúrbios de aprendizagem, como a dislexia, e o fraco desempenho em vários testes auditivos centrais ${ }^{8,9}$. Relacionando-se os achados dos testes centrais aos dados de casos com lesões detectadas por exames de neuroimagem é possível determinar a sensibilidade e a especificidade dos testes centrais ${ }^{6}$.

O objetivo deste estudo consiste em apresentar e co rrelacionar os resultados da avaliação do PA e exame de imagem (SPECT) em um grupo de indivíduos disléxicos e correlacionar os resultados do PA entre o grupo de disléxicos e o grupo de indivíduos sem queixa de aprendizagem.

\section{MÉTODO}

Participaram desta pesquisa 36 crianças, sendo 30 do sexo masculino e 6 do sexo feminino, na faixa etária de 8 a 12 anos. As crianças foram divididas em dois grupos distintos, sendo o grupo experimental (GE) formado por 18 crianças com diagnóstico de dislexia fornecido pelo Ambulatório de Neuro-Dificuldades de Aprendizagem da Faculdade de Ciências Médicas (FCM) da Universidade Estadual de Campinas (UNICAMP) e o grupo comparação (GC) composto por 18 crianças sem queixas de leitura e escrita e p a readas em relação a sexo, lateralidade e escolaridade ao GE . Todas as crianças eram destras, alunas de escola pública, de nível sócio-econômico baixo, sem déficits visuais, auditivos, cognitivos e neurológicos e todas com autorização para pesquisa por parte dos responsáveis.

As avaliações dos indivíduos participantes do estudo foram realizadas no Ambulatório de Otorinolaringologia da FCM-UNICAMP.

Inicialmente, todos foram submetidos à avaliação audiológica convencional constituída de audiometria tonal liminar, limiar de reconhecimento de fala, índice percentual de reconhecimento de fala e medidas de imitância acústica. A avaliação audiológica foi realizada em cabina acústica com o audiômetro AC-30, marca interaco ustic, calibrado no padrão ANSI-69. Para a realização da imitância acústica foi utilizado o equipamento AZ7R, como fone TDH-39 e coxim MX-41 com tom de sonda de $226 \mathrm{~Hz}$.

A seguir todos os indivíduos foram submetidos à avaliação do PA, em que foram aplicados os seguintes testes: dicótico de digítos, dicótico não-verbal, dicótico de dissílabos alternados (SSW).
O teste dicótico de dígitos foi proposto por Musiek ${ }^{10} \mathrm{e}$ adaptado para o português por Santos e Pereira ${ }^{11}$. Consiste de uma lista de 20 pares de dígitos: o paciente ouve uma seqüência de 4 dígitos por vez apresentados dicoticamente e é instruído a repetir oralmente todos os dígitos apresentados.

O teste dicótico de dissílabos alternados - proposto por Katz ${ }^{12}$ foi adaptado para o português brasileiro sob a supervisão do próprio autor por Borges, Rejtman e Scheider ${ }^{13}$. Os indivíduos são orientados para ouvir com atenção um $\mathrm{g}$ rupo de quatro palavras em seqüência, devendorepetilas na ordem de apresentação, totalizando quarenta grupos de quatro palavras. Cada palavra é apresentada a cada oreIha, havendo uma superposição parcial, isto é, a segunda sílaba da palavra inicial e a primeira sílaba da palavra final são apresentadas simultaneamente às duas orelhas. $O$ teste possibilita uma análise qualitativa de erros que são denominados de tendências de erros: tipo A, efeito auditivo, efeito de ordem e inversões.

$O$ teste dicótico não-verbal foi elaborado e descrito por Ortiz e Pereira ${ }^{14}$. $O$ teste é composto por 6 sons não verbais diferentes, sendo 3 sons onomatopéicos e 3 sons ambientais, os quais foram combinados aos pares, apresentados de fo rma simultânea em cada orelha em 3 etapas distintas. $\mathrm{Na}$ primeira etapa o paciente é solicitado a apontar em um quadro a figura correspondente a um dos dois sons apresentados auditivamente. Na etapa de atenção direita o indivíduo deve apontar somente o som apresentado à orelha d i reita e na etapa de atenção esquerda o indivíduo deve apontar somente o som apresentado à orelha esquerda. São apresentadas 24 estimulações em cada etapa, sendo os fones invertidos a cada 12 apresentações para evitar uma possível interferência da calibração dos fones.

Em um segundo momento, todos os indivíduos do GE realizaram SPECT. Os exames foram feitos pela equipe de especialistas do Serviço da Medicina Nuclear da FCM-UNICAMP. As imagens tomográficas do cére b ro foram adquiridas e reconstrúdas nos planos transversal, temporal e sagital após injeção venosa de HMPAO-99mTc.

Para a análise estatística foram utilizados: análise descritiva dos dados; para comparar dois grupos foi utilizado o teste não paramétrico de Mann-Whitney e o teste do quiquadrado; para comparar o SPECT foi utilizado o teste não paramétrico de Kruskal-Wallis; para comparar os grupos e as medidas das duas orelhas foi utilizado o ANOVA com transformação por postos (Rank) para dados pareados. $O$ mesmo teste foi utilizado para correlacionar o SPECT com os testes processados. Considerou-se o nível de significância de 0,05 .

\section{RESULTADOS}

A Tabela 1 mostra os resultados obtidos sobre o desempenho do PA na comparação entre os dois $g$ rupos (GE e GC), através da análise estatística com o teste do qui-quadrado. Observou-se que o GE apresentou diferença estatisticamente significante em relação ao GC em todos os testes do PA.

$\mathrm{Na}$ análise estatística dos resultados do teste dicó- 
Tabela 1. Correlação entre os dois grupos no teste de processamento auditivo.

\begin{tabular}{|c|c|c|c|c|c|c|}
\hline \multirow[t]{2}{*}{ Grupos } & \multicolumn{2}{|c|}{ Dicótico não-verbal } & \multicolumn{2}{|c|}{ Dicótico de dígitos } & \multicolumn{2}{|c|}{ SSW } \\
\hline & Alterado & Normal & Alterado & Normal & Alterado & Normal \\
\hline GE & 16 & 2 & 14 & 4 & 14 & 4 \\
\hline GC & 6 & 12 & 5 & 13 & 6 & 12 \\
\hline Total & 22 & 14 & 19 & 17 & 20 & 16 \\
\hline $\mathrm{p}$ & \multicolumn{2}{|c|}{0,006} & \multicolumn{2}{|c|}{0,0027} & \multicolumn{2}{|c|}{0,0073} \\
\hline
\end{tabular}

SSW, teste dicótico de dissílabos alternados.

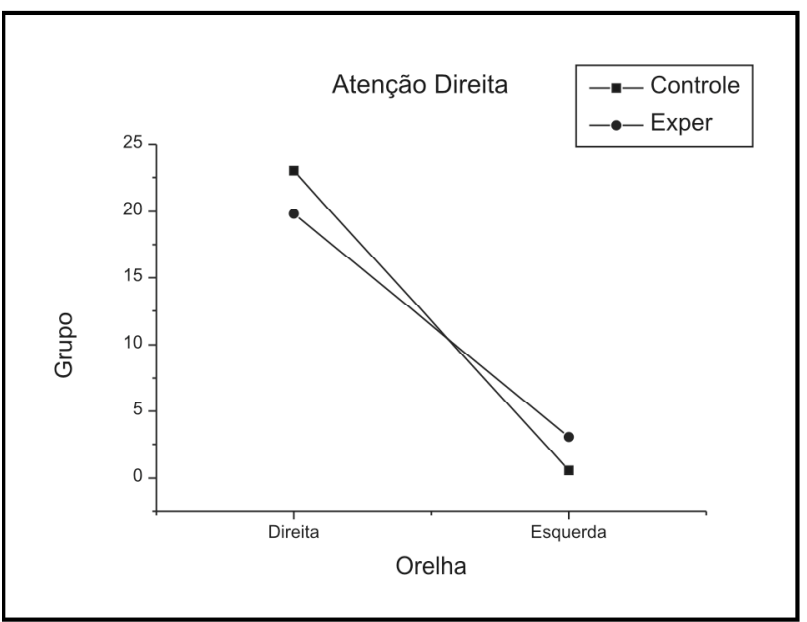

Fig 1. Houve diferença estatisticamente significante no com portamento das orelhas no teste da atenção direita entre os grupos.

tico não-verbal observamos na etapa de atenção livre índices de respostas semelhantes entre as duas oreIhas no GC, enquanto que o GE apresentou preferência à orelha esquerda. Na etapa de atenção direita o GC apresentou resultado estatisticamente significante superior à orelha direita em relação ao GE (Fig 1), pelo teste ANOVA. Na etapa de atenção esquerda não foi observada diferença estatisticamente significante entre os dois grupos estudados.

Na Tabela 2 estão expostos os resultados obtidos com o SPECT no GE. Observou-se que $50 \%$ dos exames mostraram-se alterados, sendo a maior parte ( 7 de 9 exames alterados) com hipoperfusão em áreas do lobo temporal esquerdo.

O teste não paramétrico de Kruskal-Wallis avaliou a correlação entre o PA e o SPECT e não mostrou diferença estatística nesta correlação.

\section{DISCUSSÃO}

Observando os testes do PA, encontramos diferença estatisticamente significante entre os grupos, uma vez que o GE apresentou maior número de erros. Os nossos dados não podem ser comparados com outros da literatura, pois desconhecemos estudos semelhan-
Tabela 2. Resultados obtidos com o exame de imagem (SPECT) no grupo-experimental.

\begin{tabular}{|c|c|}
\hline Sujeitos & Resultados do Spect \\
\hline 1 & Hipoperfusão na região parieto-occipital esquerda \\
\hline 2 & $\begin{array}{l}\text { Hipoperfusão da porção mesial dos lobos temporais, } \\
\text { mais acentuado à direita. }\end{array}$ \\
\hline 3 & Perfusão normal dos hemisférios cerebrais \\
\hline 4 & Perfusão normal dos hemisférios cerebrais \\
\hline 5 & Perfusão normal dos hemisférios cerebrais \\
\hline 6 & Perfusão normal dos hemisférios cerebrais \\
\hline 7 & $\begin{array}{l}\text { Hipoperfusão na porção lateral alta do lobo tem- } \\
\text { poral esquerdo }\end{array}$ \\
\hline 8 & Perfusão normal dos hemisférios cerebrais \\
\hline 9 & Perfusão normal dos hemisférios cerebrais \\
\hline 10 & $\begin{array}{l}\text { Hipoperfusão na porção mesial do lobo temporal } \\
\text { esquerdo }\end{array}$ \\
\hline 11 & $\begin{array}{l}\text { Hipoperfusão da porção mesial do lobo temporal } \\
\text { esquerdo }\end{array}$ \\
\hline 12 & $\begin{array}{l}\text { Hipoperfusão da porção inferior do lobo temporal } \\
\text { esquerdo }\end{array}$ \\
\hline 13 & $\begin{array}{l}\text { Hipoperfusão dos lobos occipitais e hemisférios } \\
\text { cerebelares }\end{array}$ \\
\hline 14 & $\begin{array}{l}\text { Hipoperfusão da porção mesial do lobo temporal } \\
\text { esquerdo }\end{array}$ \\
\hline 15 & Perfusão normal dos hemisférios cerebrais \\
\hline 16 & Perfusão normal dos hemisférios cerebrais \\
\hline 17 & $\begin{array}{l}\text { Hipoperfusão na porção anterior dos lobos tempo- } \\
\text { rais, porção inferior dos lobos frontais (esquerda) } \\
\text { e região parieto-occipital baixa à direita }\end{array}$ \\
\hline 18 & Perfusão normal dos hemisférios cerebrais \\
\hline
\end{tabular}

tes que pudessem nos fornecer dados superponíveis aos obtidos com o presente trabalho. As alterações encontradas indicam, segundo $\mathrm{Katz}^{6}$, dificuldades na o rganização de eventos sonoros no tempo, dificuldade na memória auditiva de curto prazo e dificuldade em bloquear sons competitivos. Pestun ${ }^{3}$ a firma que a memória seqüencial íntegra é imprescindível para o êxito da integração fonema-grafema e conseqüentemente interfere com a leitura que depende da lem- 
brança da ordem temporal dos fonemas e da ordem espacial dos grafemas.

Em relação ao teste dicótico de dígitos, ambos os $\mathrm{g}$ rupos apresentaram resultados melhores no canal auditivo direito do que no canal auditivo esquerdo, porém, o GE apresentou resultados acentuadamente mais rebaixados na orelha direita do que o GC. Esses achados vão de encontro à literatura, em que há relatos de menor índice de acertos ou ausência de vantagem da orelha direita em testes dicóticos na população de disléxicos ${ }^{15-17}$.

Smith e Griffiths ${ }^{18}$ utilizaram teste com sons não verbais de forma dicótica em um grupo de disléxicos e verificaram resultados idênticos aos encontrados em nosso estudo. A dificuldade do grupo de indivíduos disléxicos em realizar a assimetria perceptual na etapa de atenção direita indica dificuldade na atenção seletiva e sustentada, podendo também ser um fator predisponente para falta de habilidade em decifrar o código não verbal da comunicação ${ }^{19}$.

A teoria estrutural proposta por Kimura ${ }^{20}$ afirma que sob audição dicótica os elementos neurais da via contralateral são ativados, enquanto ocorre supressão da atividade da via ipsilateral. Essa proposta pode justificar os resultados encontrados em nosso estudo, em que o grupo de indivíduos com hipoperfusão de lobo temporal esquerdo apresentou resultados rebaixados à orelha contralateral (orelha direita).

Nas últimas décadas as técnicas de imagem têm possibilitado investigações e observações mais profundas sobre os substratos neuroanatômicos nas dificuldades de aprendizagem. Utilizando exames como a ressonância magnética vários autores encontraram em grupos de disléxicos ectopias e microgirias, entre outras alterações corticais em áreas importantes para a audição como a fissura peri-sylviana, áreas de associação do lobo temporal esquerdo, giro angular, giro supramarginal e córtex temporal posterior ${ }^{21-27}$.

Pestun ${ }^{3}$ refere que para a realização de uma leitura com compreensão adequada é necessária a integridade do sistema nervoso periférico e central, bem como certos pré-requisitos fundamentais como atenção seletiva e sustentada, discriminação e percepção auditiva, memória de curto e longo prazo e consciência fonológica, habilidades essas avaliadas no conjunto de testes especiais do PA.

Concluímos que crianças com dislexia apresentam alterações do processamento neurológico central que podem ser detectadas tanto em testes específicos de $p$ rocessamento auditivo, quanto em exames funcionais de imagem como SPECT.

\section{REFERÊNCIAS}

1. Alvarez A, Zaidan E. Processamento auditivo central: novas abordagens em habilitação. In Dislexia, céreb ro, cognição e aprendizagem. São Paulo: Frôntis Editorial, 2000.

2. Hamburger SD, Aquino T, King C, Pikus A, Cohen RM. Normal activation of frontotemporal language cortex in dyslexia, as measured with oxygen 15 positron emission tomography. Arch Neurol 1994;51:27-38.

3. Pestun MSV. Análise funcional discriminativa em dislexia do desenvolvimento. Tese Universidade Estadual de Campinas. Campinas, 2002.

4. Paulesu E, Frith U, Snowling M, et al. Is developmental dyslexia a disconnection syndrome? Evidence from PET scanning. Brain 1996;119: 143-157.

5. Rumsey JM, Nace K, Donohue B, Wise D, Maisog J, An d reason P. A positron emission tomographic study of impaired wordrecognition and phonological processing in dyslexic men. Arch Neurol 1997;54: 562-573.

6. Katz J, Ivey RG. Tratado de audiologia clínica. São Paulo: Manole, 1999.

7. American Speech Hearing and Language Association Task Force on Central Auditory Processing Consensus Development. Central auditory p rocessing: current status of research and implications for clinical practice. Am J Audiol 1996;5:41-54.

8. Musiek FE, Kibbe K, Baran JA. Neuroaudiological results from splitbrain patients. Sem Hear 1984;5:219-229.

9. Welsh L, Welsh J, Cooper B. Cortical, subcortical and brainstem dysfunction: correlation in dyslexic children. Ann Otol Rhino Laryngol 1982;91:310-315.

10. Musiek F. Assessment of central auditory dysfunction: the dichotic digit test revisited. Ear Hear 1983;4:79-83.

11. Pereira LD, Santos MFC. Escuta com dígitos. In Processamento auditivo central: manual de avaliação. São Paulo: Lovise, 1997.

12. Katz, J. The use of SSW for assessing the integrity of the central auditory nervous system. J Audit Res 1962;2:327-337.

13. B o rges ACC. Dissílabos alternados - SSW. In Processamento auditivo central: manual de avaliação. São Paulo: Lovise, 1997.

14. Ortiz KZ, Pereira LD. Teste não-verbal de escuta direcionada. In Processamento auditivo central: manual de avaliação. São Paulo: Lovise, 1997.

15. Heiervang E, Hugdahl K, Steinmetz H, et al. Planum temporale, planum parietale and dichotic listening in dyslexia. Neuropsychologia 2000;38: 1704-1713.

16. Hugdahl K, Helland T, Faerevaag MK, et al. Absence of ear advantage on the consonant-vowel dichotic listening test in adolescent and adult dyslexics: specific auditory-phonetic dysfunction. J Clin Exp Neuropsychol 1995;17:833-840.

17. Hugdahl $\mathrm{K}$, Heiervang E, Nordby $\mathrm{H}$, et al. Central auditory processing, MRI morphometry and brain laterality: applications to dyslexia. Scand Audiol 1998;49(Suppl ):26-34.

18. Smith K, Griffiths P. Defective lateralized attention for non-verbal sounds in developmental dyslexia. Neuropsychologia 1987;25:259-268.

19. Alvarez AMMA, Balen SA, Misorelli MI, Sanchez ML. Processamento auditivo central: proposta de avaliação e diagnóstico diferencial. In Munhoz MSL, Covilla HH, Silva MLG, Ganança MM (Eds.). Audiologia clínica. São Paulo: Atheneu, 2000.

20. Kimura, D. A note on cerebral dominance in hearing. Acta Otolaryngol 1963;56:617-618.

21. Galaburda AM, Cestnick L. Dislexia del desarrollo. Suplemento de Neurologia 2003;36(Suppl. 1):S3-S9.

22. Horwitz P, Rumsey JM, Donohue BC. Functional connectivity of the angular in normal reading and dyslexia: neurobiology. Proc Natl Acad Sci USA 1998:95.

23. Pauleasu E, Fritz U, Snowling M, et al. Is developmental dyslexia a disconnection syndrome? Brain 1996;119:143-157.

24. Duara R, Kushch A, Gross-Glenn K, et al. Neuroanatomic differences between dyslexic and normal readers on magnetic resonance imaging scans. Arch Neurol 1991;48:410-416.

25. Hynd GW, Hall J, Novey ES, et al. Dyslexia and corpus callosum morphology. Arch Neurol 1995;52: 32-37.

26. Rumsey JM, Donohue BC, Brady DR, et al. A magnetic resonance imaging study of planum temporale asymmetry in men with developmental dyslexia. Arch Neurol 1997;54:1481-1489.

27. Rumsey JM, Horwitz B, Donohue BC, et al. A functional lesion in developmental dyslexia: left angular gyral blood flow predicts severity. Brain Lang 1999;70:187-204. 